\title{
Study of the Band Gap Changes in Flat Acenes
}

\author{
Maryam Mazdarani ${ }^{1}$, Ali Asghar Khakpoor ${ }^{2, *}$, Bahare Agahi Keshe ${ }^{3}$ \\ ${ }^{1}$ Department of Mathematics, West Tehran Branch, Islamic Azad University, Iran \\ ${ }^{2}$ Department of Physics, Central Tehran Branch, Islamic Azad University, Iran \\ ${ }^{3}$ Young Researchers and Elite Club, West Tehran Branch, Islamic Azad University, Iran
}

Copyright $\subseteq 2019$ by authors, all rights reserved. Authors agree that this article remains permanently open access under the terms of the Creative Commons Attribution License 4.0 International License

\begin{abstract}
In the future molecular electronic is substitute the silicon electronic by the organic molecules. Due to $\mathrm{p}$ orbital's, upper and lower electron clouds, and resonance phenomenon, the aromatic hydrocarbon from the root of benzene can provide suitable environments for electron transition. A useful approach for this purpose is an appropriate pattern to predict electronic properties by topological indices method (TIM). Therefore, it is first tried to produce a relationship between the topological indices based on the number of rings; then $\mathrm{F}$ index values are measured for Circumacenes family. The gap energy changes in the family of $\mathrm{C}_{8(\mathrm{n}+2) / 3} \mathrm{H}_{(2 \mathrm{n}+22) / 3}$ was calculated using Gaussian 09 Software by Hartree-Fock method. The relationships described the gap energy changes in the Circumacenes family achieved by $\mathrm{F}$ index. Finally, some heavier members of Circumacenes family are exposed to TIM to predict of Gap energy.
\end{abstract}

Keywords Nanoelectronic, Circumacenes, HOMO-LUMO, Topological Indices Method

\section{Introduction}

Focusing on the molecular electronics is a considerable point which should attract attention because minifying the size of electronic components on Nano-scale confronts various limitations. The molecular electronics is a branch of science based on Nano-technology with multiple applications in electronic industry in which organic molecules are basic, and the electronic circuits and logic gates are designed out of the joining of these hydrocarbons [1- 4]. Aromatic hydrocarbon from the root of benzene can provide suitable environments for electron transition due to p orbital's, upper and lower electron clouds, and resonance phenomenon. A family of organic molecules which is focused in molecular electronic is Circumacenes with the chemical formula $\mathrm{C}_{8(\mathrm{n}+2) / 3} \mathrm{H}_{(2 \mathrm{n}+22) / 3}[5,6]$.

The chemical graph theory is a branch of mathematical chemistry which we would use it to solve molecular problems by the applications of graph theory. In general, a graph is used to represent a molecule by considering the atoms as the vertices of the graph and the molecular bonds as the edges. In graph-theoretical terms via the molecular graph a single number representing a chemical structure and it's called a topological descriptor. In addition if this descriptor was correlated with a property of molecule it is a topological index [7-12]. Topological indices are interesting since they capture some of the properties of a molecule in a single number. Hundreds of topological indices have been introduced and studied for modelling physical and chemical properties [13-21]. Because they are converted into optimal semiconductors by reducing band gap, the Circumacenes family are could replace to silicon semiconductors in electronic circuits in the nanoelectronics industry. Thereby, this leads to reducing the cost of manufacturing, and the size and weight of electronic circuits. For example: in field effect transistors, solar cells, sensors and etc $[22,23]$.

\subsection{Definitions}

Let $\mathrm{G}$ be a simple graph with $\mathrm{n}$ vertices and $\mathrm{m}$ edges, with vertex set $V(G)=\left\{v_{1}, v_{2}, \ldots v_{n}\right\}$ and edge set $E(G)$. The edge connecting the vertices $\mathrm{v}_{\mathrm{i}}$ and $\mathrm{v}_{\mathrm{j}}$ will be denoted by ij. The degree of the vertex $v_{i}$, denoted by $d_{i}$, is the number of first neighbours of $v_{i}$ in the graph. Followed by the first and second Zagreb indices, Furtula and Gutman were defined the forgotten topological index (or F-index) as below [24, 25]: 


$$
F=F(G)=\sum_{u \in V(G)} \operatorname{deg}(v)^{3}=\sum_{u v \in E(G)}\left[\operatorname{deg}(u)^{2}+\operatorname{deg}(v)^{2}\right]
$$

where $\operatorname{deg}(\mathrm{v})$ and $\operatorname{deg}(\mathrm{u})$ are the degrees of vertices $\mathrm{v}$ and $\mathrm{u}$ in $\mathrm{G}$ for any $v \in V(G)$.

\subsection{Hartree-Fock (HF) Method}

The HF method is based on providing the wave function for many particles systems, which the basis is the wave functions of one atom [26, 27]. In this case, the interaction Hamiltonian of many-body systems as follows:

$$
H=\sum_{i}^{N} \frac{P_{i}^{2}}{2 m_{i}}+V_{i}+V_{i j}
$$

Where $\frac{P_{i}^{2}}{2 m_{i}}, V_{i}$ and $V_{i j}$ are Kinetic energy, external interaction potential, and interaction of the electron electron respectively. The external interaction potential is given by:

$$
V_{i}=-\sum_{k=1}^{N} \frac{Z_{k} e^{2}}{\left(r_{i}-R_{k}\right)}
$$

Where $r_{i}$ is the coordinates of the ith electrons and $Z_{k}$ is an atomic number of the nucleus which is in the coordinates of $R_{k}$. And the interaction of the electron electron is given by:

$$
V_{i j}=\sum_{i<j}^{N} \frac{1}{\left(r_{i}-r_{j}\right)}
$$

\section{Research Method}

To present a simple model based on graph theory to predict electronic and optical properties of Circumacenes, it is first tried to produce a relationship between the topological indices based on the number of rings, and then $F$ index values are calculate for some members of Circumacenes family. Figure 1 shows the simple graph of Circumacenes family $(\mathrm{C}(8 \mathrm{n} 16) / 3 \mathrm{H}(2 \mathrm{n} 22) / 3)$ as.

Theorem 1: Suppose $\mathrm{n}$ is the number of dual rings in Circumacenes family; therefore $\mathrm{F}$ index equals to:

$$
F=\frac{100 n+104}{3}
$$

Proof: Consider a simple molecular graph which is divided into nine regions and shown in figure 2 :

I. All vertices and edges that are located between two L1 and L2 levels and it is called G1.

II. All vertices and edges that are located between two L2 and L3 levels and it is called G2.

III. All vertices and edges that are located between two L3 and L4 levels and it is called G3.

IV. All vertices and edges that are located between two L4 and L5 levels and it is called G4.

V. All vertices and edges that are located between two L5 and L6 levels and it is called G5.

VI. All vertices and edges that are located between two L6 and L7 levels and it is called G6

VII. All vertices and edges that are located between two L7 and L8 levels and it is called G7. 


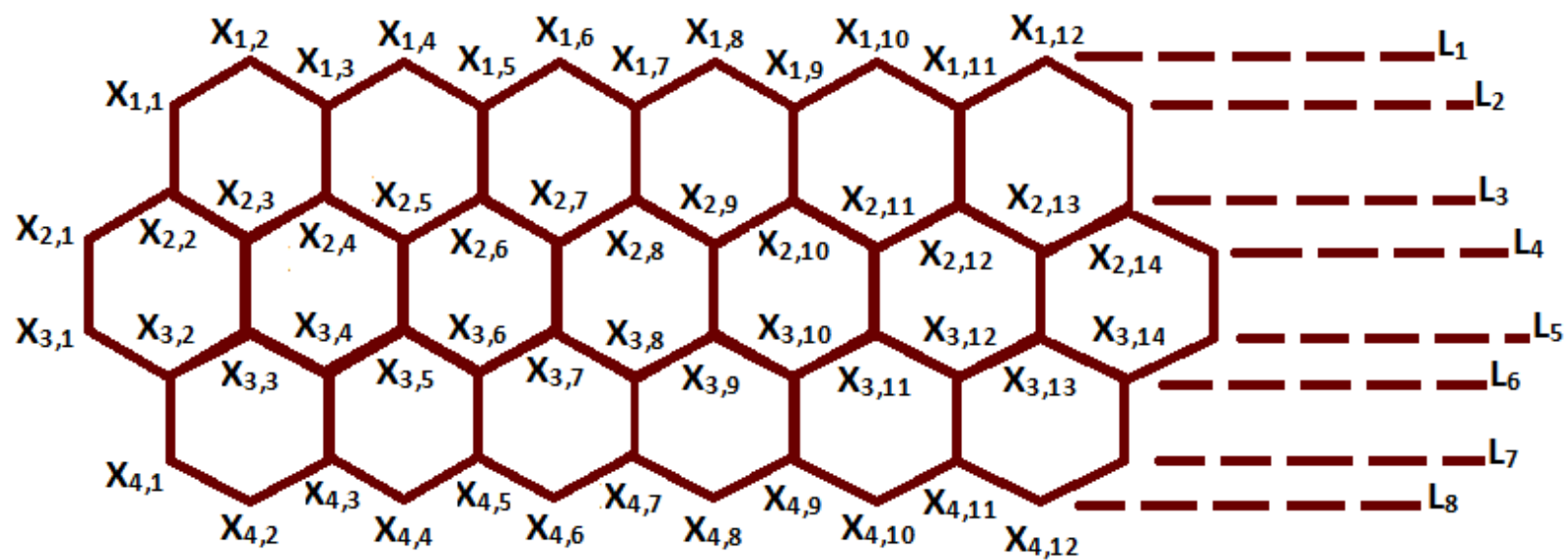

Figure 1. Simple molecular graph of Circumacenes $\left(\mathrm{C}_{(8 n+16) / 3} \mathrm{H}_{(2 \mathrm{n}+22) / 3}\right)$
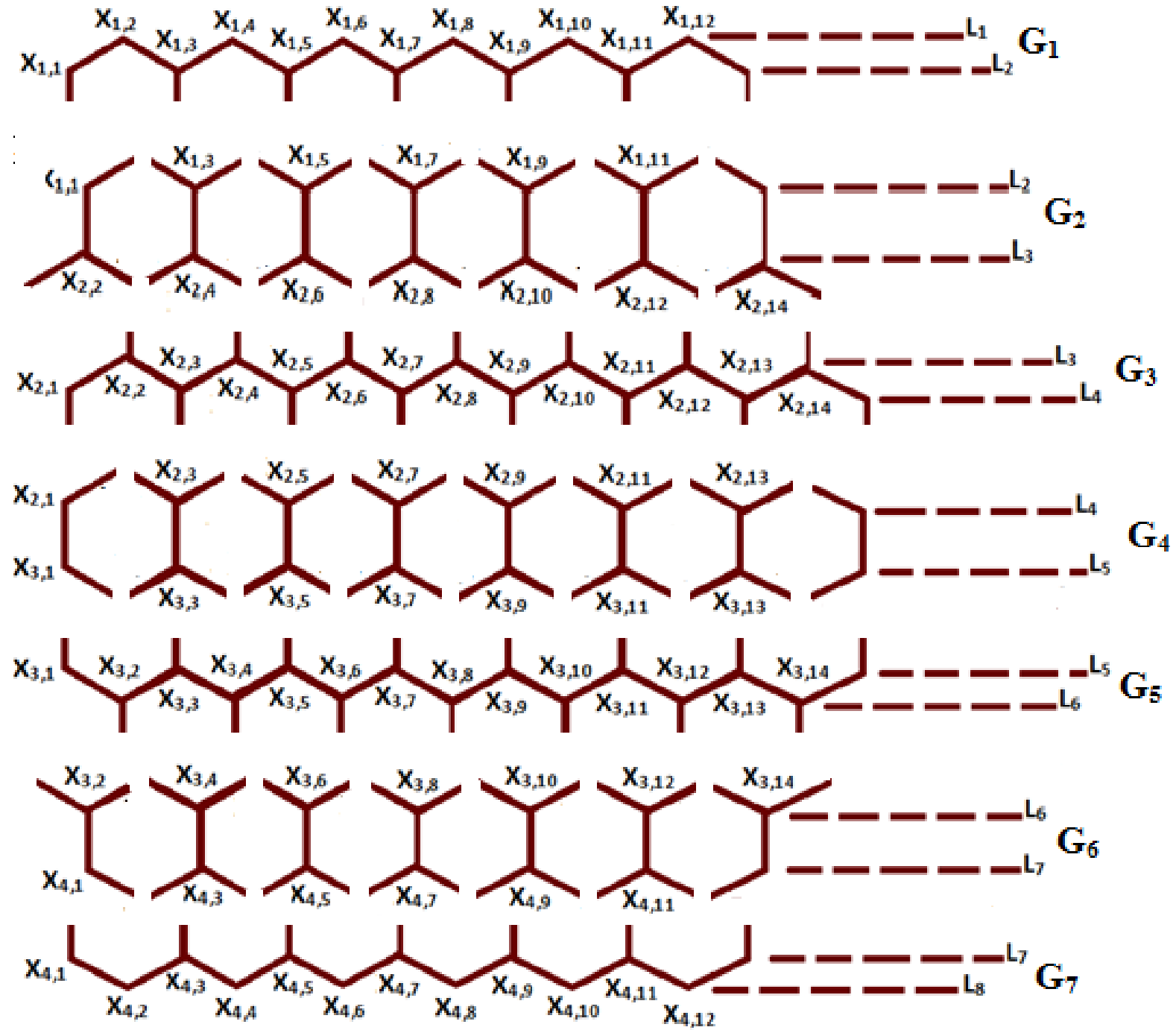

Figure 2. All vertices and edges that are located between two $\mathrm{L}_{\mathrm{i}}$ and $\mathrm{L}_{\mathrm{i}+1}(\mathrm{i}=1, \ldots, 7)$ levels 
For $\mathrm{G}_{1}$, using equation 1 , we have:

$$
F\left(G_{1}\right)=\sum_{m=1}^{k-1}\left[\left(d_{1, m}\right)^{2}+\left(d_{1, m+1}\right)^{2}\right]
$$

The first and last terms are separated:

$$
F\left(G_{1}\right)=\left(d_{1,1}{ }^{2}+d_{1,2}{ }^{2}\right)+\left(d_{1, k-1}{ }^{2}+d_{1, k}{ }^{2}\right)+\sum_{m=2}^{k-1}\left(d_{1, m}{ }^{2}+d_{1, m+1}{ }^{2}\right)
$$

And for the first two terms in equation 7 we have

$$
\left(d_{1,1}^{2}+d_{1,2}^{2}\right)=\left(d_{1, k-1}^{2}+d_{1, k}^{2}\right)=8
$$

And for $m \neq 1$ and $m \neq k$ we will have:

$$
\left(d_{1, m}^{2}+d_{1, m+1}^{2}\right)=13
$$

And though equations 8 and 9, we can conclude:

$$
F\left(G_{1}\right)=8+8+\sum_{m=2}^{k-2} 13=16+13(k-3)
$$

And since $k=\frac{2 n+1}{3}$, therefore:

$$
F\left(G_{1}\right)=16+13\left(\frac{2 n-8}{3}\right)
$$

In $G_{2}$, first we separate first and last terms:

$$
\begin{aligned}
& F\left(G_{2}\right)=\sum_{m=1}^{k}\left(d_{1, m}^{2}+d_{2, m}^{2}\right)=\left(d_{1,1}^{2}+d_{2,1}^{2}\right)+ \\
& \left(d_{1, k}^{2}+d_{2, k}^{2}\right)+\sum_{m=2}^{k-1}\left(d_{1, m}^{2}+d_{2, m}^{2}\right)
\end{aligned}
$$

Equation 12 signifies for the first two terms as:

$$
\left(d_{1,1}^{2}+d_{2,1}^{2}\right)=\left(d_{1, k}^{2}+d_{2, k}^{2}\right)=13
$$

And for two terms of $\mathrm{m} \neq 1$ and $\mathrm{m} \neq \mathrm{k}$, we have:

$$
\left(d_{1, m}^{2}+d_{2, m}^{2}\right)=8
$$

Equations 13 and 14 will lead to:

$$
F\left(G_{2}\right)=13+13+\sum_{m=2}^{k-1} 8=26+8(k-2)
$$

And $k=\frac{n+2}{3}$, so:

$$
F\left(G_{2}\right)=26+8\left(\frac{n-4}{3}\right)
$$

In $G_{3}$, first we separate first and last terms: 
$F\left(G_{3}\right)=\sum_{m=1}^{k-1}\left(d_{2, m}^{2}+d_{2, m+1}^{2}\right)=\left(d_{2,1}^{2}+d_{2,2}^{2}\right)+\left(d_{2, k-1}{ }^{2}+d_{2, k}{ }^{2}\right)+\sum_{m=2}^{k-2}\left(d_{2, m}^{2}+d_{2, m+1}^{2}\right)$

Equation 17 signifies for the first two terms as:

$$
\left(d_{2,1}^{2}+d_{2,2}^{2}\right)=\left(d_{2, k-1}^{2}+d_{2, k}^{2}\right)=13
$$

And for two terms of $m \neq 1$ and $m \neq k$, we have:

$$
\left(d_{2, m}^{2}+d_{2, m+1}^{2}\right)=8
$$

Equations 18 and 19 will lead to:

$$
F\left(G_{3}\right)=13+13+\sum_{m=2}^{k-2} 8=26+8(k-3)
$$

And $k=\frac{2 n+7}{3}$, so:

$$
F\left(G_{3}\right)=26+8\left(\frac{2 n-2}{3}\right)
$$

For $\mathrm{G}_{4}$, we can write:

$$
F\left(G_{4}\right)=\sum_{m=1}^{k}\left(d_{2, m}^{2}+d_{3, m}^{2}\right)
$$

The first and last terms are separated:

$$
\begin{aligned}
& F\left(G_{4}\right)=\left(d_{2,1}{ }^{2}+d_{3,1}{ }^{2}\right)+\left(d_{2, k}{ }^{2}+d_{3, k}{ }^{2}\right) \\
& +\sum_{m=2}^{k-1}\left(d_{2, m}{ }^{2}+d_{3, m}{ }^{2}\right)
\end{aligned}
$$

And for the first two terms in equation 23 we have

$$
\left(d_{2,1}^{2}+d_{3,1}^{2}\right)=\left(d_{2, k}^{2}+d_{3, k}^{2}\right)=1
$$

And for $\mathrm{m} \neq 1$ and $\mathrm{m} \neq \mathrm{k}$ we will have:

$$
\left(d_{2, m}^{2}+d_{3, m}^{2}\right)=8
$$

And though equations 24 and 25, we can conclude:

$$
F\left(G_{4}\right)=8+8+16+8(k-2)
$$

And $k=\frac{n+5}{3}$, therefore:

$$
F\left(G_{4}\right)=2+8\left(\frac{n-1}{3}\right)
$$

The calculation procedure of $\mathrm{G}_{5}, \mathrm{G}_{6}$ and $\mathrm{G}_{7}$ are as the same as $\mathrm{G}_{3}, \mathrm{G}_{2}$, and $\mathrm{G}_{1}$ respectively and is given through equations 11, 16 and 21, Therefore:

$$
F(G)=F\left(G_{1}\right)+F\left(G_{2}\right)+F\left(G_{3}\right)+F\left(G_{4}\right)+F\left(G_{5}\right)+F\left(G_{6}\right)+F\left(G_{7}\right)
$$


So, equation 5 is achieved and the theorem is proved.

Then $\mathrm{F}$ index was calculated for some Circumacenes family members using equation 5 and the results are shown in Table 1.

Table 1. F index for the first five members of Circumacenes family

\begin{tabular}{|c|c|c|}
\hline Chemical Formula & IUPAC Name & F Index \\
\hline $\mathbf{C}_{\mathbf{2 4}} \mathbf{H}_{\mathbf{1 2}}$ & coronene & 268 \\
\hline $\mathbf{C}_{\mathbf{3 2}} \mathbf{H}_{\mathbf{1 4}}$ & ovalene & 368 \\
\hline $\mathbf{C}_{\mathbf{4 0}} \mathbf{H}_{\mathbf{1 6}}$ & circumanthracene & 468 \\
\hline $\mathbf{C}_{\mathbf{4 8}} \mathbf{H}_{\mathbf{1 8}}$ & circumtetracene & 568 \\
\hline $\mathbf{C}_{\mathbf{5 6}} \mathbf{H}_{\mathbf{2 0}}$ & circumpentacene & 668 \\
\hline
\end{tabular}

\section{Results}

The band gap energy $\left(\mathrm{E}_{\mathrm{gap}}\right)$ of Circumacenes family $(\mathrm{C} 8(\mathrm{n}+2) / 3 \mathrm{H}(2 \mathrm{n}+22) / 3)$ was calculated using Gaussian 09 Software and Hartree-Fock (HF) method. The results were compared to the experimental data and from the validated references [22,23]. These results were given in Table 2 .

Table 2. The band gap energy $\left(E_{g a p}\right)$ of the first five members of Circumacenes family

\begin{tabular}{|c|c|}
\hline Chemical Formula & $\boldsymbol{E}_{\text {gap }}(\boldsymbol{e V})$ \\
\hline $\mathbf{C}_{\mathbf{2 4}} \mathbf{H}_{\mathbf{1 2}}$ & 3.42 \\
\hline $\mathbf{C}_{\mathbf{3 2}} \mathbf{H}_{\mathbf{1 4}}$ & 2.69 \\
\hline $\mathbf{C}_{40} \mathbf{H}_{\mathbf{1 6}}$ & 2.03 \\
\hline $\mathbf{C}_{\mathbf{4 8}} \mathbf{H}_{\mathbf{1 8}}$ & 1.54 \\
\hline $\mathbf{C}_{\mathbf{5 6}} \mathbf{H}_{\mathbf{2 0}}$ & 1.18 \\
\hline
\end{tabular}

Figure 3 shows the band gap energy changes for Circumacenes family according to $\mathrm{F}$ index as the results of this work.

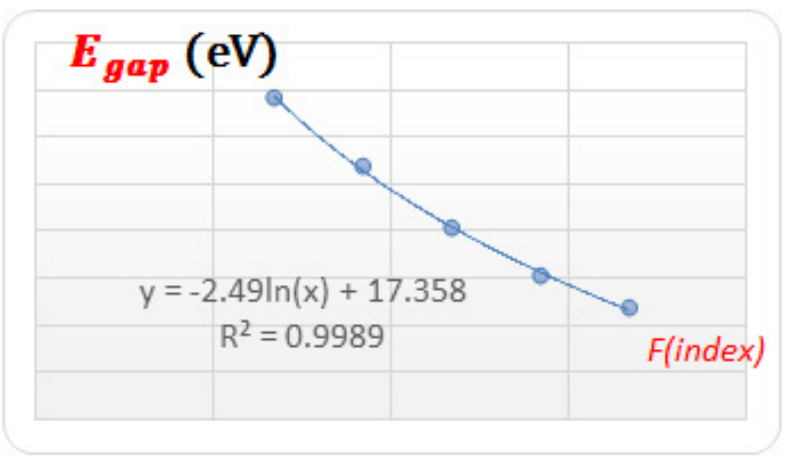

Figure 3. The band gap energy changes in Circumacenes family according to $\mathrm{F}$ index

Figure 3 shows that the band gap be calculated by the $\mathrm{F}$ index with a high level of precision ( $2=0.9989)$, as below:

$$
E_{\text {gap }}=-2.49 \ln (F)+17.358
$$

\section{Conclusions}

For providing the validity of TIM method the band gap changes were calculated in five members of Circumacenes family using equation 26, and the results shows in Table 3. These results could be compared with the reference values (Table 2).

Table 3. Calculation the band gap of five members Circumacenes through TIM method

\begin{tabular}{|c|c|}
\hline Chemical Formula & $\mathbf{E}_{\text {gap }}(\mathbf{e V})$ \\
\hline $\mathbf{C}_{24} \mathbf{H}_{12}$ & 3.4364 \\
\hline $\mathbf{C}_{32} \mathbf{H}_{14}$ & 2.6468 \\
\hline $\mathbf{C}_{40} \mathbf{H}_{16}$ & 2.0483 \\
\hline $\mathbf{C}_{48} \mathbf{H}_{18}$ & 1.5661 \\
\hline $\mathbf{C}_{56} \mathbf{H}_{20}$ & 1.1623 \\
\hline
\end{tabular}

In heavier members of Circumacenes family which the experimental and other theoretical methods are too difficult and very expensive, the TIM method is an optimized method. So, the equation 26 was used to calculation the band gap in three heavier members of Circumacenes family and results shows in Table 4.

Table 4. Predicting Gap Energy through TIM method

\begin{tabular}{|c|c|c|}
\hline Chemical Formula & F Index & $\mathbf{E}_{\text {gap }}(\mathbf{e V})$ \\
\hline $\mathbf{C}_{\mathbf{6 4}} \mathbf{H}_{\mathbf{2 2}}$ & 768 & 0.8149 \\
\hline $\mathbf{C}_{72} \mathbf{H}_{\mathbf{2 4}}$ & 868 & 0.5101 \\
\hline $\mathbf{C}_{\mathbf{8 0}} \mathbf{H}_{\mathbf{2 6}}$ & 968 & 0.2386 \\
\hline
\end{tabular}

With increasing the number of the atoms in the heavier members of this family, the energy levels of conduction bands and capacity also has increased. So, the highest occupied molecular orbital (HOMO) is shifted to up and also the lowest unoccupied molecular orbital (LUMO) shifted to down at the same time. As a result the band gap has decreased and the electron transition due to the $p$ orbital's, upper and the lower electron clouds have increased in the heavier members of Circumacenes.

\section{REFERENCES}

[1] Vito Sgobba and Dirk M. Guldi,Carbon nanotubes - electronic/electrochemical properties and application for nanoelectronics and photonics, Chem. Soc. Rev. 38 (2009) 165-184.

[2] Patolsky F, Timko BP, Zheng G, Lieber CM. Nanowire-based nanoelectronic devices in the life sciences. MRS Bulletin; Univ Press Cambridge, 2007.

[3] Pérez-Jiménez AJ, Sancho-Garca JC. Conductance Enhancement in Nanographene-Gold Junctions by Molecular $\pi$-Stacking. J. Am. Chem. Soc. 131, (2009) 14857-14867. 
[4] Sancho-García JC, Pérez-Jiménez AJ. Charge-Transport Properties of Prototype Molecular Materials for Organic Electronics Based on Graphene Nanoribbons. Phys Chem Chem Phys. 11(2009) 2741-2746.

[5] Jiang D, Dai S. Circumacenes versus Periacenes: HOMO-LUMO Gap and Transition from Nonmagnetic to Magnetic Ground State with Size, Chem. Phys. Lett. 466 (2008) 72-75.

[6] Lias S, Ionization Energy Evaluation. Lindstrom PJ, Mallard WG. Eds., NIST Chemistry Web-Book, NIST Standard Reference Database Number 69. the U.S. Secretary of Commerce on behalf of the United States of America: National Institute of Standards and Technology; 2005.

[7] Ivanciuc $\mathrm{T}$, Ivanciuc $\mathrm{O}$, Klein DJ. Modeling the bio concentration factors and bioaccumulation factors of polychlorinated biphenyls with posetic quantitative super-structure/activity relationships (QSSAR). Mol Divers. 10(2) (2006) 133-145.

[8] Bytautas L, Klein DJ. Chemical Combinatorics for Alkane-Isomer Enumeration and More. J. Chem. Inf. Comput. Sci. 47(6) (2007) 2124-2132.

[9] Nikolić S, Miličević A, Trinajstić N. QSPR Study of Polarographic Half-wave Reduction Potentials of Benzenoid Hydrocarbons. Croat. Chem. Acta. 79(1) (2006) 155-159.

[10] Wiener H. Structural Determination of Paraffin Boiling Points. J. Am. Chem. Soc. 69(1) (1947) 17-20.

[11] Dobrynin A. A., Entringer R., Gutman I., Wiener index of trees: theory and applications, Acta Appl. Math. 66 (2001) 211-249.

[12] Gutman I., Konstantinova E. V., Skorobogatov V. A., Molecular hypergraphs and Clar structural formulas of benzenoid hydrocarbons, ACH Models Chem. 136 (1999) 539-548.

[13] Khakpoor A. A., Agahi Keshe B., Siamy B., The Relation between Total $\pi$ Electron Energy of Linear Acenes as Nanostructures and Topological Indices, J. Comput. Theor.Nanosci. 12(12) (2015) 5617-5621.

[14] Khakpoor A. A., Agahi Keshe B., Agahi Kesheh A., Nano Structures and Its Thermodynamic Properties Using TIM, Journal of Materials Science and Chemical Engineering 3 (11) (2015) 1-6.

[15] Khakpoor A. A., Agahi Keshe B., Electronic and Optical Properties of Nanostructures and Its Relationship with Harari Index, Journal of Materials Science and Chemical Engineering. 3(8) (2015) 1-5.

[16] Khakpoor A. A., Agahi Keshe B., Physical and Electro-Optical Properties of Rylenes as Nanostructures Using Topological Indices Method, Journal of Nanoelectronics and Optoelectronics. 11(3) (2016) 280-283.

[17] Gholamhossein F., Khakpoor A. A., Agahi Keshe B., Mazdarani M., Study of Electron Affinity and Gap Energy in Electronic Nanostructures, Science of Advanced Materials. 9 (7) (2017) 1120-1125.

[18] Khakpoor A. A, Prediction Electronic and Physical
Properties of Nano Structures; Topological Index Method, INTERNATIONAL JOURNAL OF ADVANCED RESEARCH,3(6)(2015)1536-1540

[19] Khakpoor A. A, Agahi Keshe B, Some Electronic and Optical Properties of Nanostructures Using the First Zagreb Index, INTERNATIONAL JOURNAL OF ADVANCED RESEARCH,3(5)(2015)548-551

[20] Khakpoor A. A., Agahi Keshe B, DETERMINATION OF PHYSICAL-CHEMICAL PROPERTIES OF TITANIUM ORGANIC COMPOUNDS USING TOPOLOGICAL INDICES; QSPR TECHNIQUES, INTERNATIONAL JOURNAL OF CURRENT RESEARCH,7(5)(2015)15686 $-15689$

[21] Khakpoor A. A, Total $\pi$ Electron Energy of Linear Acenes Nanostructure, International Letters of Chemistry, Physics and Astronomy,64(2016)110-115

[22] Kadantsev ES, Stott MJ, Rubio A. Electronic Structure and Excitations in Oligoacenes from ab Initio Calculations, J. Chem. Phys. 124 (2006) 134901.

[23] Malloci G, Mulas G, Cappellini G, Joblin C. Time-Dependent Density Functional Study of the Electronic Spectra of Oligoacenes in the Charge States -1, $0,+1$, and +2 , Chem. Phys. 340(1-3) (2007) 43-58.

[24] Gutman I, Furtula B, Elphick C. Three New/Old VertexDegree-Based Topological Indices, MATCH Commun. Math. Comput. Chem. 72 (2014) 617-632.

[25] Vuki cevi'c D, Furtula B., Topological index based on the ratios of geometrical andarithmetical means of end-vertex degrees of edges, J. Math. Chem. 46 (2009) 1369-1376.

[26] Sakurai J. J. Advanced Quantum Mechanics, Addison-Wesley, Boston, USA, 1967.

[27] David J. Griffiths, Introduction to Quantum Mechanics, 2nd Ed.; Addison-Wesley, Boston, USA, 2004. 